\title{
Consensus formation on adaptive networks
}

\author{
Balazs Kozma ${ }^{1}$ and Alain Barrat ${ }^{1,2}$ \\ ${ }^{1}$ LPT, CNRS, UMR 8627, and Univ Paris-Sud, Orsay, F-91405 (France) \\ ${ }^{2}$ Complex Networks Lagrange Laboratory, ISI Foundation, Turin, Italy
}

(Dated: October 27, 2018)

\begin{abstract}
The structure of a network can significantly influence the properties of the dynamical processes which take place on them. While many studies have been devoted to this influence, much less attention has been devoted to the interplay and feedback mechanisms between dynamical processes and network topology on adaptive networks. Adaptive rewiring of links can happen in real life systems such as acquaintance networks where people are more likely to maintain a social connection if their views and values are similar. In our study, we consider different variants of a model for consensus formation. Our investigations reveal that the adaptation of the network topology fosters cluster formation by enhancing communication between agents of similar opinion, though it also promotes the division of these clusters. The temporal behavior is also strongly affected by adaptivity: while, on static networks, it is influenced by percolation properties, on adaptive networks, both the early and late time evolution of the system are determined by the rewiring process. The investigation of a variant of the model reveals that the scenarios of transitions between consensus and polarized states are more robust on adaptive networks.
\end{abstract}

PACS numbers: 89.75.-k, -87.23.Ge, 05.40.-a

\section{INTRODUCTION, MOTIVATION}

The recent past has witnessed an important development of the activities of statistical physicists in the area of social sciences, motivated by the fact that statistical physics is the natural field to study how global complex properties can emerge from purely local rules. Statistical physics models and tools have therefore been applied to the understanding of issues related to the characterization of the collective social behavior of individuals, such as culture dissemination, the spreading of linguistic conventions, and the dynamics of opinion formation [1].

The statistical physics approach tries to grasp the essential features of emerging social behaviors, and considers therefore simple rules of opinion formation in which agents update their internal state, or opinion, through an interaction with their neighbors. According to the "herding behavior" described in sociology [2, 3], such interaction typically consists of agents following local majority [4, 5, 6] or imitating a neighbor [7]. Starting from random initial conditions, and without any global supervision, the system self-organizes through an ordering process possibly leading to the emergence of a global consensus, in which all agents share the same opinion. Alternatively, the system can reach a state of polarization, in which a finite number of groups with different opinions survive, or of fragmentation, with a final number of opinions scaling with the system size.

In certain models, opinions are represented very schematically by a discrete variable which can take two values (0 or 1$)$, similarly to Ising spins; this is the case in the Voter model 7], for which, at each timestep, an agent is chosen at random and adopts the opinion of one of its neighbors. Some additional realism on the modeling of opinions is put forward in Axelrod's model [8], where opinions or culture are represented by a vector of cultural traits. Features such as memory may also be introduced, with interesting new emerging behaviors 9, 10, 11, 12, 13]. Another refinement with respect to the use of binary opinions is introduced in the Deffuant model [14] where opinions are continuous variables (see also [15, 16, 17, 18]). The latter models also introduce the notion of bounded confidence: an agent will interact with another agent only if their opinions are close enough. The bounded confidence is described by a tolerance parameter, and the system can evolve towards different states of polarization depending on the value of this parameter.

As a first natural step, many studies of such simple models have considered that each agent was allowed to interact with all the others. This mean-field-like scenario can indeed be realistic when dealing with a small number of agents. Moreover, the case of agents embedded into low-dimensional lattices has as well been a topic of interest. Recently however, the growing field of complex networks [19, 20, 21, 22, 23] has allowed to obtain a better knowledge of social networks [24, 25], and in particular to show that the typical topology of the networks on which social agents interact is not regular. Various studies have therefore considered the evolution of the aforementioned models when agents are embedded on more realistic networks, and studied the influence of various complex topologies on the corresponding dynamical behavior (see for example [26, 27, 28, 29]).

Up to now, few studies have however considered the fact that many networks have a dynamical nature, and that their evolution occurs on a timescale which may have an impact on the dynamical processes occurring between the nodes. Such considerations are particularly relevant for social network which continuously evolve, a priori on various timescales (both fast and slow). Moreover, the evolution of the topology and the dynamical processes 
can drive each other with complex feedback effects. The topology may indeed have an impact on the evolution of the agents' states, which in its turn determines how the topology can be modified [30, 31, 32, 33, 34, 35, 36]: the network becomes adaptive.

In this paper, we therefore investigate how the coevolution of an adaptive network of interacting agents and of the agents' opinions influence each other, and how the final state of the system depends on this coevolution. We focus on the Deffuant model for which a large number of opinions can coexist (and not only 2 as in the Voter Model). Moreover, and in contrast with most other studies of evolving networks, the rate of evolution of the network's topology is tunable and represents one of the parameters of the model. We focus on simple evolution rules that do not require prior knowledge of the state of agents to which new links are established. We investigate the role of the various parameters such as the tolerance of agents and the rate of topology evolution. We show that the possibility of the interaction network to adapt to the changes in the opinion of the agents has important consequences on the evolution mechanisms and on the structure of the system's final state.

The organization of the paper is as follows: In Section III the Deffuant model and the investigated quantities are defined. In order to have a full description of the model, we start our study with the opinion formation of static networks in Section III. Then, the case of adaptive networks is considered in Section[IV] in comparison with the static case. In this section, we study the effects of the rewiring on the final state of the consensus formation. Next, in Section $\nabla$ the investigation of the temporal behavior of the system gives us a deeper understanding of the processes taking place of adaptive networks. Finally, in Section VI, a variant of the original Deffuant model is considered on static and adaptive networks.

\section{DEFINITION OF THE MODEL AND QUANTITIES OF INTEREST}

The model we consider is based on the Deffuant model for interacting agents [14]. In this model, $N$ agents $(i=1, \cdots, N)$ are endowed with a continuous opinion $o$ which can vary between 0 and 1 and is initially random. Two agents, $i$ and $j$ can a priori communicate with each other if they are connected by a link, i.e. if they are neighbors. At each time step $t$, two neighboring agents are selected, and they communicate if their opinions are close enough, i.e., if $|o(i, t)-o(j, t)| \leq d$, where $d$ defines the tolerance range or threshold. In this case, the (local) communication tends to bring the opinions even closer, according to the rule

$$
\begin{aligned}
& o(i, t+1)=o(i, t)+\mu(o(j, t)-o(i, t)) \\
& o(j, t+1)=o(j, t)-\mu(o(j, t)-o(i, t))
\end{aligned}
$$

where $\mu \in[0,1 / 2]$ is a convergence parameter. For the sake of simplicity we will consider the case of $\mu=1 / 2$ which corresponds to $i$ and $j$ adopting the same intermediate opinion after communication [15]. The role of the tolerance threshold has been characterized in the mean-field topology where all agents are neighbors of each other. For large tolerance values, agents can easily communicate and converge to a global consensus. On the contrary, small values of $d$ naturally lead to the final coexistence of several remaining opinions.

In the present study, we consider more realistically that agents have a limited number of neighbors. The initial interaction network structure is taken as an uncorrelated random graph in which agents have $\bar{k}$ acquaintances on average, i.e. the initial network corresponds to an ErdösRényi network with average degree $\bar{k}$.

In the next section, we will study for reference the case of a static interaction network. This framework considers that the topology of the agents' interaction does not evolve, or evolves at a rate which is infinitely slow with respect to the communication between agents. It is however also interesting to consider the fact that social interactions may evolve on the same time scale as agents' opinions, and possibly in a way depending on these opinions. Agents indeed may break, keep or establish connections according to how much frustration or reward they get from the corresponding relationship. The network along which communication and possible convergence of opinions occur becomes then time-dependent. Many possibilities can be thought of for modeling this time evolution: links may for example decay at constant rate, independently from the agents' opinions [36]. Within the framework of opinion dynamics with bounded confidence however, it seems natural to consider that only connected agents having opinions which differs more than the tolerance range may decide to terminate the relationship. In order to keep the average number of interactions constant, a new link is then introduced between one of the agents having lost a connection and another agent. In our model, this new link is established at random [42]. Naturally, this new link can break again if the newly connected agents have too far apart opinions. The rewiring process thus occurs as a random search for agents with close enough opinions.

The model therefore considers two coexisting dynamical processes: local opinion convergence for agents whose opinions are within the tolerance range, and rewiring process for agents whose opinions differ more. The relative frequencies of these two processes is quantified by the parameter $w \in[0,1]$. The precise rules of evolution are therefore summarized as follows. At each time step $t$, a node $i$ and one of its neighbors $j$ are chosen at random. With probability $w$, an attempt to break the connection between $i$ and $j$ is made: if $|o(i, t)-o(j, t)|>d$, a new node $k$ is chosen at random and the link $(i, j)$ is rewired to $(i, k)$ [43]. With probability $1-w$ on the other hand, the opinions evolve according to (11) if they are within the tolerance range. If $w>0$, the dynamics stops when no link connects nodes with different opinions. This can correspond either to a single connected network in which 
all agents share the same opinion, or to several clusters representing different opinions. For $w=0$ on the other hand, the dynamics stops when neighboring agents either share the same opinion or differ of more than the tolerance $d$.

Using a semi-formal algorithmic description let us rewrite the steps of the simulation:

1. Choose a node randomly, node $i$.

2. Pick one of its neighbors, node $j$.

3. Generate a random number, $r$, between 0 and 1 .

4. if $(r>w)$

$$
\begin{aligned}
& \text { then if }(|o(i, t)-o(j, t)|<d) \\
& \text { then opinion convergence for } i \text { and } j: \\
& o(i, t+1)=o(i, t)+\mu(o(j, t)-o(i, t)) \\
& o(j, t+1)=o(j, t)-\mu(o(j, t)-o(i, t))
\end{aligned}
$$

fi

else if $(|o(i, t)-o(j, t)|>d)$

then update the link between $i$ and $j$ :

(a) Choose a random node, node $k$, which is neither $i$ nor $i$ 's neighbor.

(b) Break the link between $i$ and $j$ then connect $i$ with $k$.

fi

fi

\section{Start again from Step 1.}

The evolution of the system and its final state can be characterized by the investigation of the opinion clusters of agents. In the final state, such clusters are made of agents sharing the same opinion. During the dynamical evolution however, many agents have close but not identical opinions, so that we generalize the concept of opinion clusters in the following way: two agents are considered as members of the same opinion cluster if there is a path of agents in between them on the interaction network where each consecutive agent in the path has an opinion within the tolerance value of the previous agent. This corresponds to the idea that there is a channel of communication in between them to share ideas. The notion of opinion clusters gives a natural way to keep track of the structure of the system over the whole dynamical process.

In the case of evolving (adaptive) networks, we also keep track of the topological clusters which correspond simply to the various connected components of the network. In the final state, the topological and opinion cluster naturally coincide, while for static networks, a unique connected cluster of agents can host several opinion clusters.

Before turning to a detailed analysis of the model, we illustrate in Fig.s[1]and[2 the different behaviors observed for static and adaptive networks. The figures show the

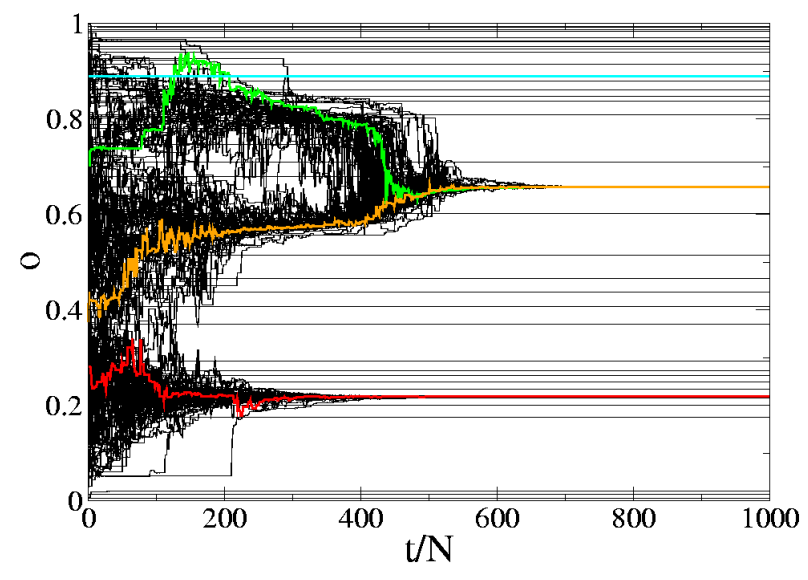

FIG. 1: (Color online) Evolution of the opinions of $25 \%$ of the population, denoted by lines, for a system of $10^{3}$ agents with tolerance $d=0.15$ and average degree $\bar{k}=5$, on a static network for a single run. The evolution of the opinion of a few individuals is highlighted with color.

evolution of the opinions of 250 out of $N=1000$ agents as a function of time, in each case for one single realization of the dynamics with $d=0.15$. The opinions are initially randomly distributed on the interval $[0,1]$. When the interaction network is static, local convergence processes take place and lead to a large number of opinion clusters in the final state, with few macroscopic size opinion clusters and many small size groups: agents with similar opinions may be distant on the network and not be able to communicate. This is in contrast with the mean-field case in which all agents are linked together so that the final opinion clusters are less numerous and more separated in the opinion space. Figure 2, which corresponds to an adaptive network with $w=0.7$, is strikingly in contrast with the static case: no small groups are observed. We will investigate these differences in more details in the next sections.

In particular, the whole cluster-size distribution gives a complete description of the system. Interesting summaries are given by the number of clusters, the size of the largest and second-largest opinion-cluster which will tell us about the behavior of the clusters with macroscopic size (because of the possible large number of small clusters, the average size may be biased towards small values and is therefore of less interest).

\section{CONSENSUS FORMATION ON STATIC NETWORKS}

Let us first consider for reference the Deffuant model on a static Erdős-Rényi network. Figure 3 displays the average relative size of the largest $\left(\left\langle S_{\max }\right\rangle / N\right)$ and 


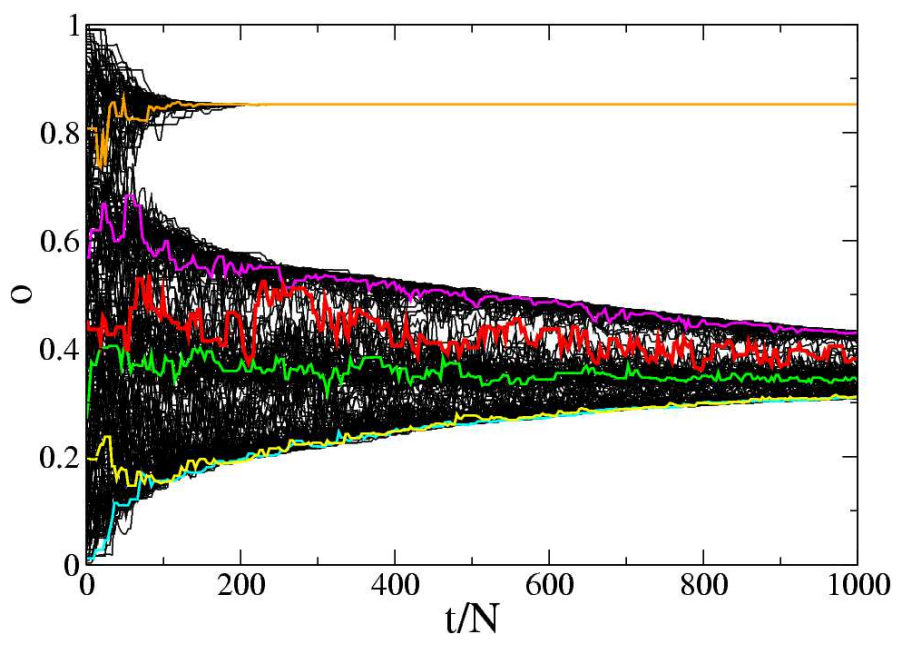

FIG. 2: (Color online) Same plot as for Fig. 1 for an adaptive network when the rate of update is $w=0.7\left(N=10^{3}, \bar{k}=5\right.$, and $d=0.15)$.

second-largest opinion clusters $\left.\left(\left\langle S_{2}\right\rangle / N\right)\right)$ in the final state, as a function of the tolerance parameter $d$, for various system sizes. Simulations are averaged over 100 different networks. Three different phases can be readily identified. At large tolerance values $d>d_{1}$, the system is in a consensus state, with a single macroscopic-size cluster present in the final state. A jump of $\left\langle S_{\max }\right\rangle / N$ from a value close to 1 to a value close to $1 / 2$ is observed around $d_{1} \approx 0.256$, together with the appearance of a macroscopic second largest cluster. This jump becomes sharper and sharper when the system size increases, hinting at a first-order phase transition in the thermodynamic limit.

The evolution of the number of opinion clusters in the final state, $N_{\text {clust }}$, sheds more light on the system's behavior, as shown in Fig. 4. For $d>0.5$, only one final cluster containing all nodes is obtained. As $d$ decreases, $\left\langle S_{\max }\right\rangle / N$ decreases very slowly while $\left\langle S_{2}\right\rangle / N$ remains close to 0 and $N_{\text {clusters }}$ increases: a large number of small clusters of finite size appear. When $d$ approaches and crosses the transition point, an interesting non-monotonic behavior is observed: $N_{\text {clusters }}$ decreases as the tolerance of the agents decreases towards $d_{1}$. This corresponds to the appearance of a second largest cluster of large size. This second large cluster contains agents with opinion $o_{2}$ different from the largest cluster's opinion $o_{1}$. The "global" tolerance range of these two large clusters is therefore wider than if only one large cluster of agents with the same opinion is present: it goes from $\left[o_{1}-d, o_{1}+d\right]$ to the union $\left[o_{1}-d, o_{1}+d\right] \cup\left[o_{2}-d, o_{2}+d\right]$ and therefore allows to communicate with more agents and obtain less small (finite size) clusters in the final state. The larger this second cluster is, the less the finite-size clusters remain isolated, therefore $N_{\text {clusters }}$ decreases.

For $d<d_{1}$, an apparently polarized state is entered, with a first and second-largest clusters of similar exten-

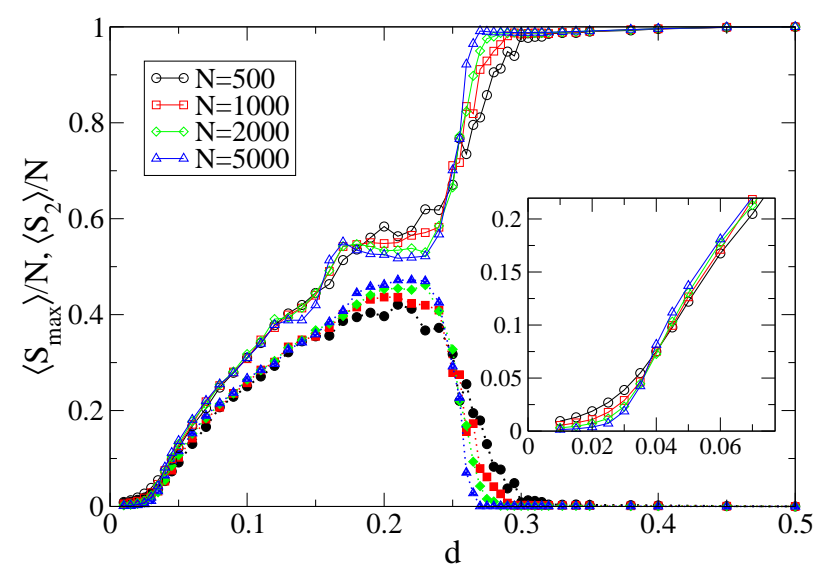

FIG. 3: (Color online) Size of the largest (empty symbols) and second-largest (filled symbols) clusters in the final state, as a function of the tolerance value on a static Erdös-Rényi network with average degree $\bar{k}=10$ for different system sizes. The color coding is the same for the first and second-largest cluster with respect to the system size. Inset: Same plot for the largest cluster zoomed into small tolerance values near the polarized-fragmented transition.

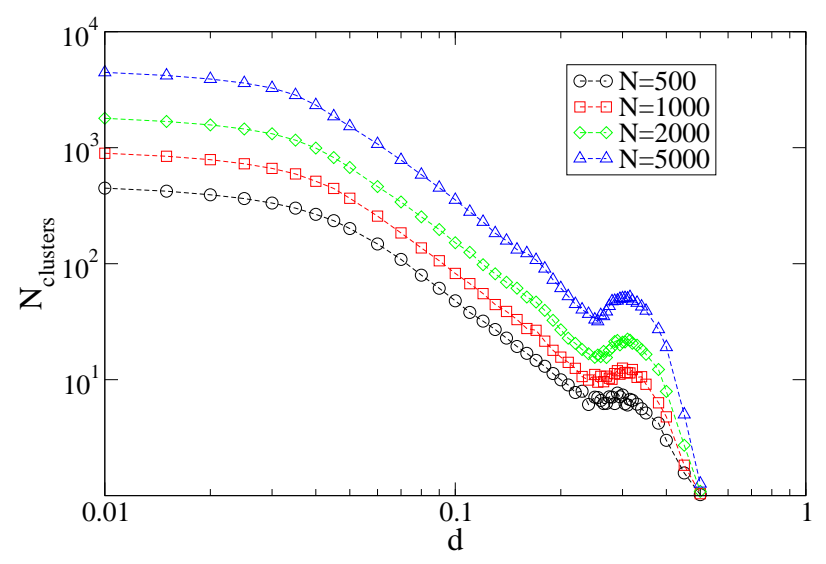

FIG. 4: (Color online) Number of clusters in the final state as a function of the tolerance of the agents for different system sizes on a static Erdős-Rényi network with average degree $\bar{k}=10$.

sive sizes. The investigation of the number of clusters (Fig. (4) however shows that the system is in a "false"polarized state, in which the number of clusters increases with the system size. This state therefore consists of a coexistence of macroscopic opinion clusters with an extensive number of finite size clusters. As $d$ decreases, the decrease of the sizes of the largest and second-largest clusters is thus due to two reasons: the appearance of more and more macroscopic-size clusters, as it is also the case in mean-field, and the proliferation of finite size "microscopic" clusters. This last point is made more explicit by the investigation of the whole cluster-size distribution 
displayed in Fig. 5 for $d=0.1$. The figure shows that the distribution of normalized sizes $s=S / N$ is composed of two parts

$$
\rho_{N}^{0}(s) \approx \delta(s) f^{0}(N)+Q^{0}(N, s),
$$

where $f^{0}(N) \propto N$ gives the number of isolated small clusters and $Q^{0}$ is a regular part describing clusters of macroscopic size.

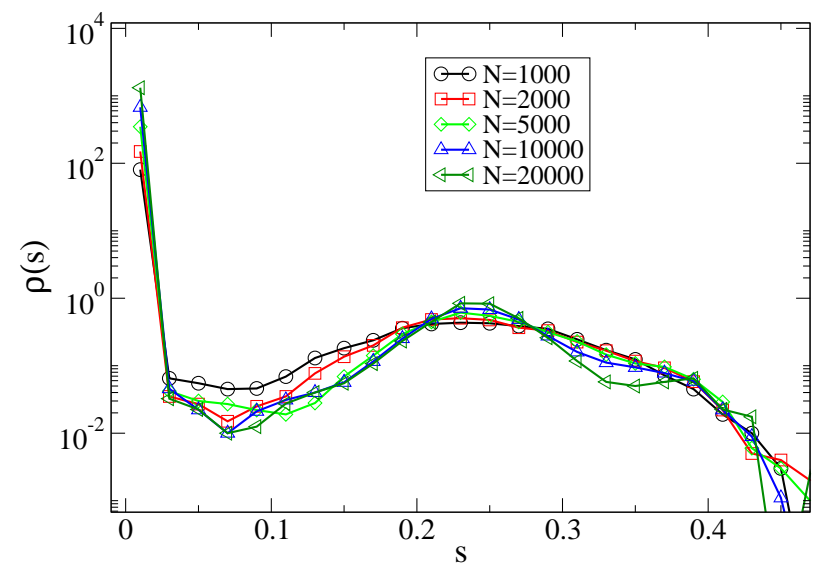

FIG. 5: (Color online) Cluster size distribution $\rho(s)$ for $d=$ 0.1 and $\bar{k}=10$ on a static Erdős-Rényi network with average degree $\bar{k}=10$.

As $d$ is even further decreased, $\left\langle S_{\max }\right\rangle / N$ vanishes for $d<d_{2}$ in the thermodynamic limit, as shown in the inset of Fig. 3. The final state of the system is then fragmented with a number of clusters saturating at $N$ as $d \rightarrow 0$ (see Fig. (4). This polarized-fragmented transition stems from the finite connectivity of the agents and is akin to a percolation transition. It is indeed due to the fact that, if the tolerance is too small, the probability for an agent to find another neighboring agent with whom to interact vanishes; the communication paths thus disappear from the network. Let us consider an agent $i$ with $k$ connections. The probability that a given neighbor has initially an opinion within the tolerance range is simply $2 d$ so that the average number of neighbors with whom he can communicate is $2 d k$. The condition for the existence of percolating paths of close enough opinions is thus simply $\bar{k}>1 /(2 d)$, and the transition to fragmentation is expected at $d_{1} \approx 1 /(2 \bar{k})$. Figure 6 displays the size of the largest cluster in the final state as a function of $d$ for various values of the average degree $\bar{k}$ of the network, showing indeed that the polarized-fragmented transition occurs at a tolerance value which scales as $1 / \bar{k}$.

We finally note that the polarized-fragmented transition is expected to disappear if the interaction network is scale-free with a diverging second moment of the degree distribution [19]. The percolation transition indeed occurs at a vanishing threshold in such networks in the thermodynamic limit. We have indeed checked (not shown) that the polarized-fragmented transition is then shifted

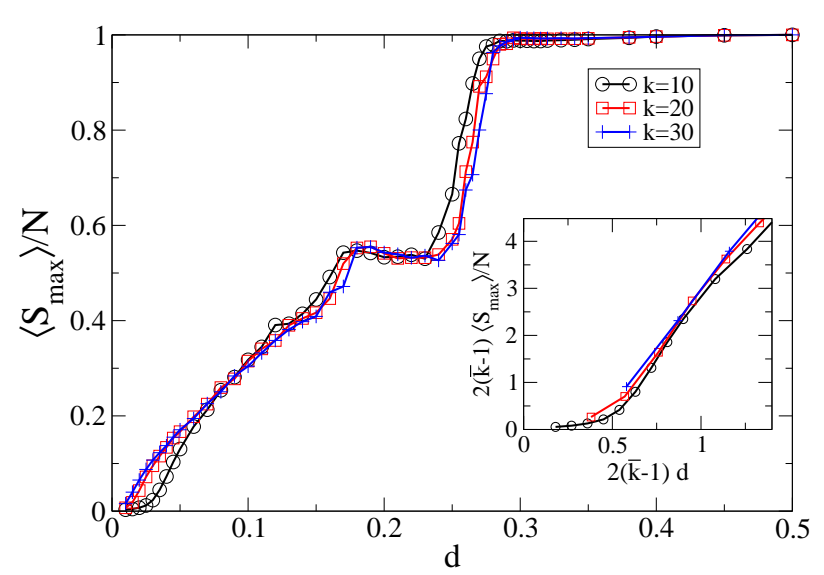

FIG. 6: (Color online) Size of the largest cluster as a function of the tolerance value on static Erdős-Rényi networks $(w=0)$ for different average connectivities. Inset: same plot, rescaled by the average connectivity and zoomed into the fragmentedpolarized transition.

to much smaller values of $d$, vanishing in the thermodynamic limit.

\section{CONSENSUS FORMATION ON ADAPTIVE NETWORKS}

Let us now turn to the case of adaptive network in which agents with far apart opinions can break their connection. The rate of attempts to rewire connections is given by $w$ : the larger $w$ and the faster rewiring can occur. Figure 7 displays the sizes of the largest and second largest clusters in the final state of the system, and Fig. 8 shows the total number of clusters.

At large enough tolerance, a unique cluster gathering all agents is obtained, as in the static case. As the tolerance decreases, a consensus-to-polarized transition is also observed, with the emergence of a second-largest cluster with extensive size at $d_{1}(w)$. The jump in $\left\langle S_{\max }\right\rangle / N$ becomes sharper as $N$ increases, indicating a first order transition as in the static case. Interestingly, we observe that $d_{1}(w)$ increases with $w$ (see inset of Fig. 7): the more easily agents can change their connections, the larger tolerance values are necessary to achieve consensus: agents can more easily search for other agents with whom they can communicate, and break ties with the ones with too different opinions, so that the formation of different clusters is favored.

As $d$ decreases below $d_{1}$, a polarized phase is observed. While the sizes of the largest and second-largest clusters are close to the case of a static network, important differences have to be noted. First of all, in the adaptive network case, each opinion cluster corresponds to a distinct connected component in the final configuration. The network is therefore broken into $N_{\text {clusters }}$ disconnected components, while the static network remained 


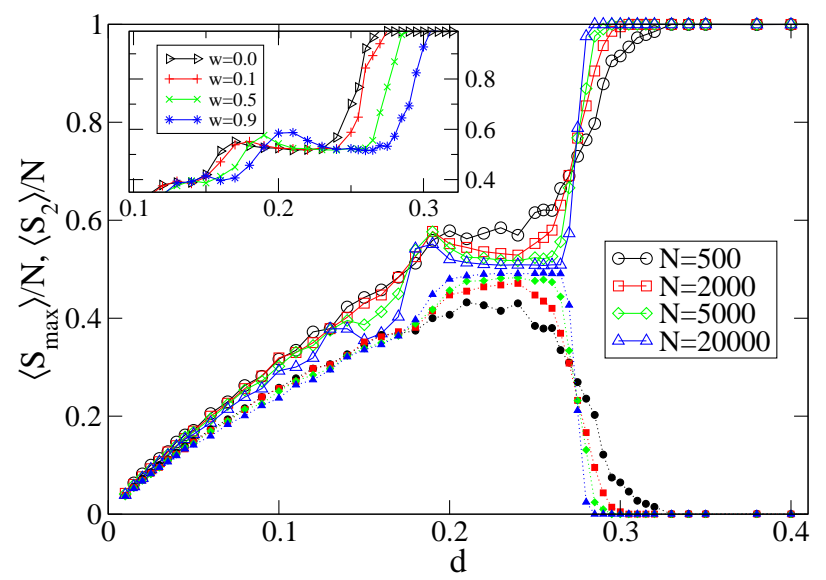

FIG. 7: (Color online) Size of the largest (open symbols) and second-largest (filled symbols) clusters as a function of the tolerance value on adaptive networks for different system sizes $(\bar{k}=10, w=0.5)$. The color coding is the same for the first and second-largest cluster with respect to the system size. Inset: maximal cluster size as a function of $d$ for different rewiring rates. The consensus-to-polarized transition point is shifted to larger and larger tolerance values as the adaptivity of the networks increases $(N=5000, \bar{k}=10)$.

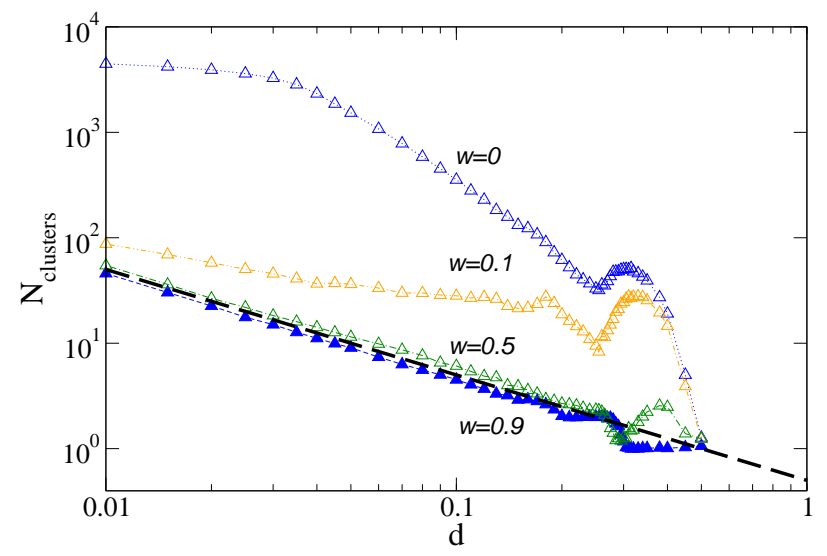

FIG. 8: (Color online) Number of clusters in the final state as a function of the tolerance of the agents for different rewiring rates on adaptive networks with average degree $\bar{k}=10$ and $N=5000$. The case of a static system $(w=0)$ is also shown for reference. The dashed line corresponds to the value $1 /(2 d)$, asymptotically valid at small tolerances in the mean-field case.

connected by definition. Moreover, the number of clusters is much smaller for adaptive than for static networks, and decreases as $w$ increases, as shown in Fig. 8 .

More insight is given by the investigation of the normalized cluster size distribution, shown in Fig. 9 for $d=0.1$ and $\bar{k}=10$. Similarly to the static case, it is formed of two parts,

$$
\rho_{N}^{w}(s) \approx \delta(s) f^{w}(N)+Q^{w}(N, s)
$$

with $f^{w}(N) \propto N^{\beta(w)}$ as shown in Fig. 10, and $Q^{w}(N, s)$ is the distribution of clusters of macroscopic size that converges to a regular finite distribution in the large $N$ li
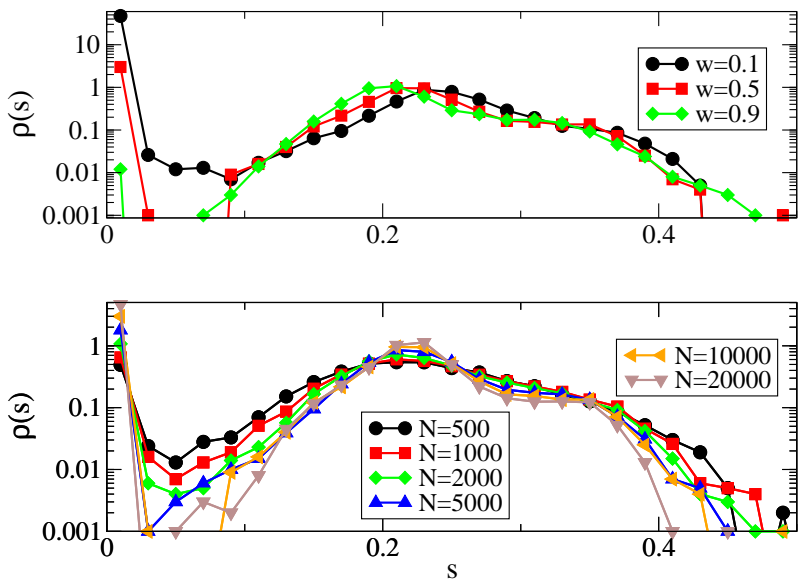

FIG. 9: (Color online) Cluster size distribution $\rho(s)$ for $d=$ 0.1 and $\bar{k}=10$, for various $w$ at $N=10^{4}$ (top) and for various sizes at $w=0.5$ (bottom).

The first part of the distribution corresponds to debris of finite-size. As can be seen in Fig. 10, on adaptive networks $(w \neq 0)$ the expected size of the clusters in the debris is increasing sublinearly with the system size, so that their weight is vanishing compared to the system size, i.e. $f^{w}(N) / N \rightarrow 0$ in the thermodynamic limit, while clusters of finite size compose a finite fraction of the system in the static case $(\beta(w=0)=1)$.

The polarized phase on adaptive networks is therefore different from the one on static networks: thanks to the possibility of link rewiring, agents who would remain isolated (or in very small groups) on a static network may manage to find agents with whom to communicate and thus enter a macroscopic cluster. Without rewiring on the other hand, a macroscopic number of agents remain in fragmented components which coexist with few macroscopic clusters.

As shown in Fig. 9 the regular part of the cluster size distribution shifts to smaller and smaller cluster sizes as the rewiring rate $w$ increases. This phenomenon is similar to the shifting of the transition point between the consensus and the polarized states: increasing the rewiring rate allows agents to find more easily other agents with whom to communicate and the formation of smaller clusters is favored.

Figure 11 finally compares adaptive and static networks for small values of the tolerance. Strikingly, the fragmented phase disappears as soon as the rewiring of the links is enabled. The size of the largest component decays smoothly as the tolerance decreases, but remains extensive, in contrast with the static case. Rewiring processes thus allow the small clusters to group together and reach extensive sizes even below the polarized-to- 


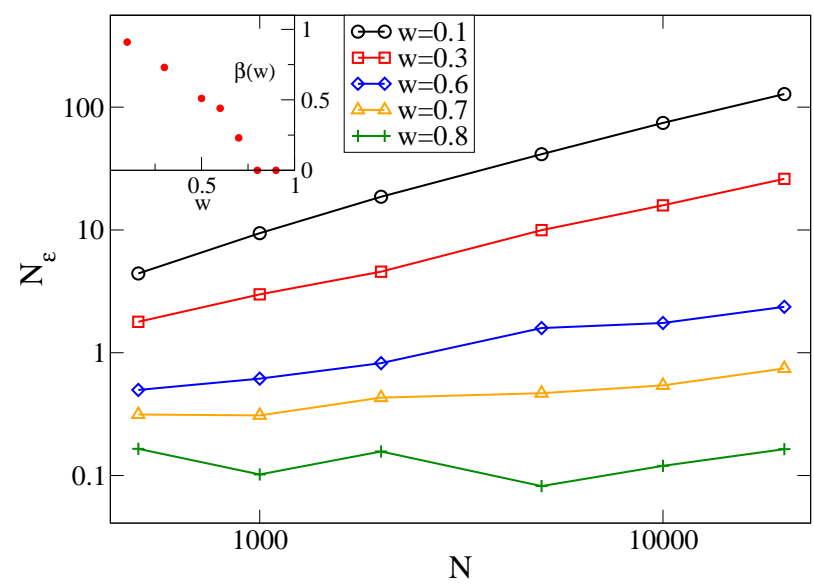

FIG. 10: (Color online) Number of clusters with size less than $\epsilon=N / 50$ for different rewiring rates in systems with $\bar{k}=10$ and $d=0.1$. Inset: measured exponents of the system-size dependent divergence of the number of clusters $\left(\propto N^{\beta(w)}\right)$ in the "debris".

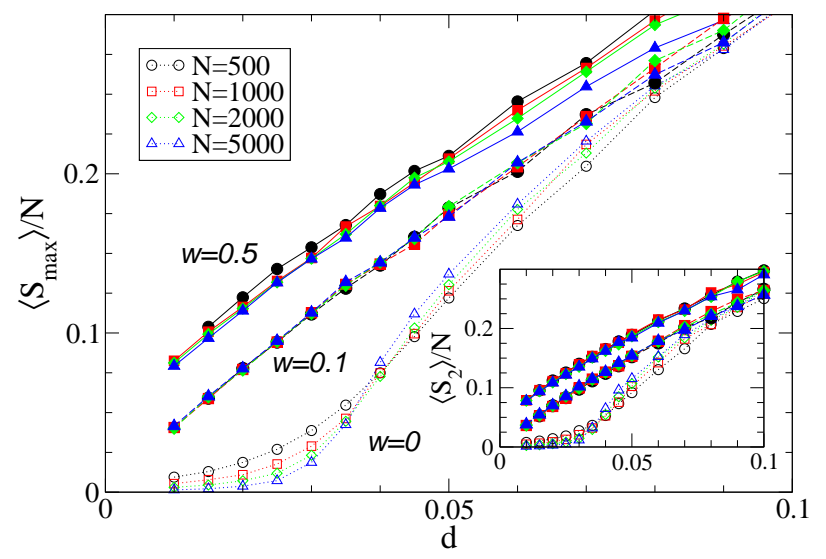

FIG. 11: (Color online) Size of the largest cluster as a function of the tolerance value on adaptive networks (filled symbols) for different system sizes $(\bar{k}=10)$, compared with the same quantity on static network (open symbols). The curves for $w=0.5$, otherwise collapsing with the $w=0.1$ ones, are shifted for clarity. Inset: same for the second-largest cluster.

fragmented transition, present only on static networks.

The comparison of the static and adaptive cases shows that the ability of the network to adapt as a consequence of the opinion dynamics has strong consequences, which interestingly are somehow opposite in the various tolerance ranges and for the various types of clusters. On the one hand, large clusters can be more easily broken by rewiring, and global consensus is more difficult to reach. At intermediate tolerance values, the extensive clusters are smaller when rewiring is enhanced. On the contrary, the small clusters of finite size have opportunities to find agents with whom to communicate, and therefore to merge with large clusters, leading to a strongly decreased total number of clusters and to a true polarized state instead of a mixture of polarization and fragmentation. At very small tolerance values finally, the fragmentation transition even disappears, and extensive clusters are obtained for arbitrarily small tolerance.

\section{TEMPORAL BEHAVIOR}

In the previous sections, we have focused on the final state in which the system settles as a result of the interplay between opinions' and network's dynamics. In order to better understand the role of the different processes, we now consider the time evolution of the clusters of agents. For the static network, only opinion clusters evolve. On the other hand, topological and opinion clusters are not identical for adaptive network: a given connected component of the network can host several opinion clusters.

First, as a reference, let us consider the temporal behavior on static networks: In this case, the time of convergence depends on the distance of $d$ from the fragmented-to-polarized transition, $d_{2}$. For large tolerance values, $d_{2}<d$, the convergence time grows linearly with the system size (data not shown) and it increases as $d \rightarrow d_{2}$ since, in this limit in the percolating opinionclusters, the average length of the communication path between two nodes increases [38] and the clusters are becoming more and more tree-like. Near $d_{2}$, the average length of the communication channel between two nodes in an opinion cluster grows as a nontrivial power of the system size [39] which in turn raises the possibility of a convergence time which grows faster than linearly with the system size (not shown). For $d<d_{2}$, the convergence time grows linearly with logarithmic corrections with respect to the system size. Though, it decreases as the tolerance of the agents decreases since the size of the tree-like opinion clusters also decreases. For very small tolerance values $\left(d \ll d_{2}\right)$, the system in fact almost does not evolve since agents rarely find neighbors with whom they can communicate.

In the case of adaptive networks, Fig. 12 shows the evolution of the number of opinion clusters $N_{O C}$ and of topological clusters (connected components) $N_{T C}$. The figures clearly show the existence of three different timescales for the clusters' evolution. In the initial configuration, a large number of separate opinion clusters are found, corresponding to percolation clusters; their number is naturally larger for smaller tolerance values and smaller $\bar{k}$. The early-time evolution is then mostly determined by the adaptive nature of the network which allows agents to look for other agents with similar opinions. The importance of this early-time behavior can be particularly emphasized in the small tolerance regime which would lead to a fragmented state for a static network. As shown in Fig. 12 indeed, the number of opinion clusters decreases very fast, from an extensive to a finite number, while the network is still globally connected 
$\left(N_{T C}=1\right)$. The possibility of rewiring connections allows therefore to form percolating/macroscopic clusters even at small tolerance values, which explains the disappearance of the fragmented phase. The characteristic time of this phenomenon is given by the time necessary for an agent to find at least one partner "to be able to communicate with," $t_{f}$. If the rewiring rate $w$ is large, $N_{O C}$ reaches a minimum before increasing again when opinions evolve. An opinion cluster indeed hosts agents that are connected by a path of potential communication, but even neighboring agents' opinions can evolve and drift further apart due to interactions with other neighbors. An opinion cluster can therefore divide itself into several clusters because of the opinion dynamics, and $N_{O C}$ increases. The corresponding timescale $t_{o}$ describes the formation of groups of agents with identical opinions on a still connected network. Finally, after the formation of groups with uniform opinions, the number of topological clusters increases and converges to the number of opinion clusters. This last phase therefore corresponds to a breaking of the links which join opinion clusters with different opinions. The timescale of this final change, $t_{l}$, characterizes the time needed by an agent to rewire its links with agents out of his tolerance range towards agents with the same opinion. Depending on the parameters of the system, this final regime can take place at timescales either much larger than those associated with the opinion cluster formation, or on similar timescales. The two possibilities are illustrated in both Fig.s 12 and 13. The case of widely separated timescales allows to consider that the opinion dynamics and rewiring process occur independently, with links evolving between fixed opinion clusters; further investigation is then possible and a mean-field analysis allows to gain insight into the clusters' topological structure [37].

The dependence of the various timescales on the parameters can be estimated as follows:

i) Initially, there is always a finite fraction of separated nodes, loners, which are not members of any percolating opinion clusters both below and above the fragmented-topolarized transition. Therefore the time characterizing the transition of $N_{O C}$ from an extensive to a finite value is $t_{f}$. A typical loner has no neighbor with whom to communicate in the initial configuration. The time needed to rewire any of its links is proportional to $1 / w$, and the probability to find an agent within tolerance range is $2 d$, so that

$$
t_{f} \propto 1 /(w d)
$$

ii) The estimation of the timescale of the opinion evolution is a more complex task. In all cases, opinions evolve at a rate $(1-w)$, so that $t_{o} \propto \tau_{o} /(1-w)$, where $\tau_{o}$ is yet to be characterized. For low rewiring rates and when $d \ll d_{2}$, the success rate of the discussions are determined by the ratio of newly found friendly neighbours and the average degree of the node. Therefore, the larger is this ratio, the smaller is $\tau_{o}$. When $d \gg d_{2}$, a typical agent can successfully communicate with the $2 d$ fraction

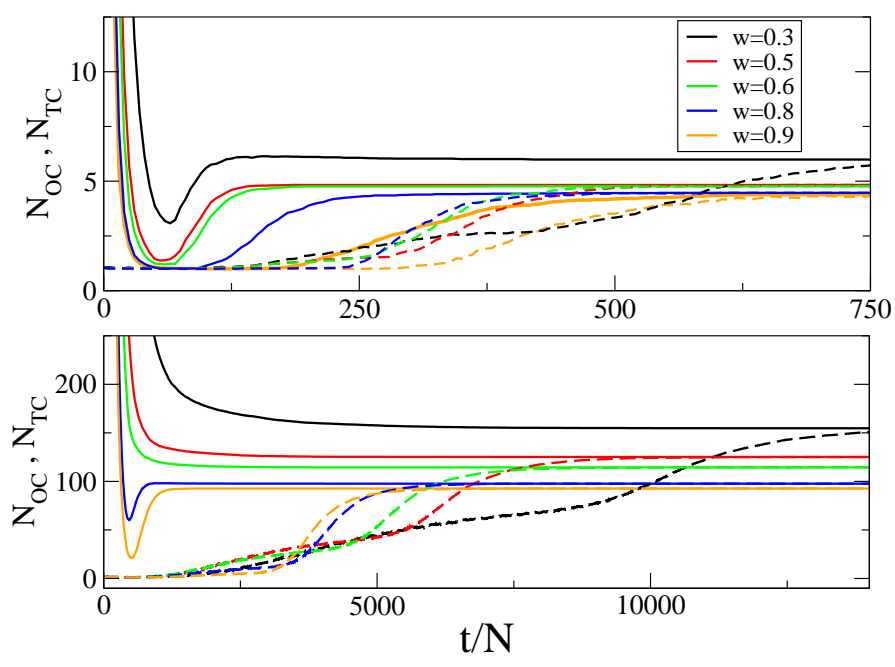

FIG. 12: (Color online) Temporal behavior of the system with high (top: $d=0.1, \bar{k}=10$, and $N=10^{3}$ ), and low (bottom: $d=0.005, \bar{k}=10$, and $N=10^{4}$ ) tolerance of the agents. (full lines: number of opinion clusters; dashed lines: number of topological clusters)

of his friends irrespectively of his degree. Therefore $\tau_{o}$ can only depend weakly on $\bar{k}$ in this case (see Fig. 13). Before considering the behavior of $t_{o}$ for high rewiring rates, the scaling of $t_{l}$ can be estimated as follows:

iii) For any $d$ values, links are updated with frequency $w$. Let us consider a typical opinion cluster. The number of its links which need to be rewired is proportional to the total number of links $(\propto \bar{k} N)$, and to the amount of opinions outside of the tolerance range $(\propto(1-2 d))$. The probability to rewire towards a close enough opinion is moreover $\propto d$ so that

$$
t_{l} \propto \bar{k}(1-2 d) /(w d)
$$

For low rewiring rates, $t_{l}$ is the longest timescale of the time evolution therefore the convergence time also scales as $t_{l}$.

For high rewiring rates, it is possible for a typical agent to successfully rewire all his links to point to agents with tolerable opinions before committing himself to changing his own opinion. This situation takes place when $t_{l}$ is less than or comparable to $1 /(1-w)$. In this case, almost all of the negotiations are successful and both the convergence time and $t_{o}$ are expected to be $\propto 1 /(1-w)$. Though, observations show that the situation is more convoluted: as the tolerance of the agents increases, fewer and fewer opinion clusters are present in the system. Nevertheless, the disappearance of a cluster in many cases happen by an initial unsuccessful attempt to form two or more separate clusters which eventually merge into one. During this initial evolution most of the links are broken between these communities and the convergence to a common opinion is only mediated by few 


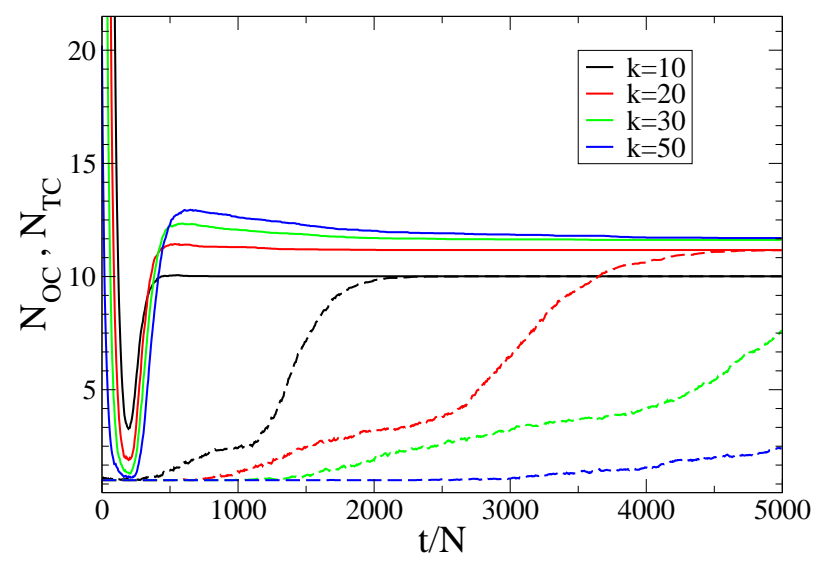

FIG. 13: (Color online) Temporal behavior of the system for fixed $w$ and $d$ and varying $\bar{k}(N=2000, w=0.5$, and $d=$ 0.05). For these parameter values, the estimation in the text predicts larger and larger separation between $t_{l}$ and $t_{o}$, which is confirmed by the simulation data. (full lines: number of opinion clusters; dashed lines: number of topological clusters)

individuals connected to both groups (see for example the red curve in Fig. 2). These individuals form a narrow channel of communication between the two communities throughout the process resulting in very long convergence times in certain regimes of tolerance values.

Similar merging of communities can be also be observed on static networks though their behavior is less drastic (see Fig. 11). Even if the merging is started by few individuals, similarly to what happens on adaptive networks, as soon as the average opinion of the two communities become close to each other, the members of the two communities suddenly engage in fruitful discussions and their opinions converge rapidly to a uniform value.

Finally, Fig. 12 illustrates how the timescale of opinion evolution increases when $w$ increases and how the separation between $t_{o}$ and $t_{l}$ increases as the tolerance $d$ is reduced, so that a change of parameters can lead from similar to well separated timescales. Figure 13 moreover shows that an increase in the average degree also leads to more and more separated timescales, as seen from the arguments in paragraph ii) and Eq. (5).

\section{A VARIANT OF THE MODEL}

The Deffuant model considers agents that have a certain tolerance range and can strictly not communicate with agents having opinions outside this range. This drastic behavior can seem unrealistic, and we consider here a variation of the model in which agents still have a finite probability to communicate event if their initial opinions are far apart. While various extensions of the update rules could be considered, we limit our study to the following simple generalization of the model: if agents $i$ and $j$ have close enough opinions, i.e., if $|o(i)-o(j)|<d$, they adopt the same intermediate opinion $\frac{o(i)+o(j)}{2}$; if $\mid o(i)-(o(j) \mid>d$ on the other hand, the opinions of the two agents converge to their mean value with probability

$$
p=e^{1-\frac{|o(i)-o(j)|}{d}} .
$$

In the exponent, the 1 term is present to make the probability, $p$, continuous when $|o(i)-o(j)|=d$. The rewiring rule is also changed: two agents may break their connection to each other only if they are outside of each others tolerance range and, in this case, they do it with probability

$$
p_{r w}=1-e^{1-\frac{|o(i)-o(j)|}{d}} .
$$

In this probabilistic model, consensus is always achieved on any static network, since any couple of neighboring agents always have some probability to communicate and reach the same opinion. Decreasing the tolerance of the agents only increases the corresponding convergence time (not shown). On adaptive networks however, the rewiring rule allows opinion clusters to separate, and a picture similar to the one of the original Deffuant model is obtained, as shown in Fig. 14, with a transition between a consensus state at large tolerance to a polarized state as $d$ decreases. The transition is also shifted to larger and larger tolerance values as $w$ increases. It is interesting to compare the effect of rewiring on the system with the original consensus-formation rules and in the case of this variant when $d<d_{2}$ : in the original model, rewiring drove the system to a more homogeneous (polarized) state than that observed on static networks (where fragmentation is obtained); while, in this variant of the model, rewiring drives the system to a more inhomogeneous state (polarized) than that on static networks (which is a consensus reached in very long times).

Interestingly, the behavior of the model on adaptive networks is in fact more robust than on static networks, since the same global picture is observed for strict or probabilistic communication rules, while a strong difference is obtained on static networks.

\section{CONCLUSIONS}

In this paper, we have studied consensus formation on static and adaptive networks through the investigation of a simple model of opinion dynamics with bounded confidence: agents with close enough opinions reach an agreement, while they can not communicate if their opinions are too far apart. When the agents are linked through a static interaction network, two transitions are found: at large tolerance values, a global consensus is reached; intermediate tolerance leads to a coexistence of several extensive groups or clusters of agents sharing a common opinion with a large number of small (finite-size) clusters. At very small tolerance values finally, a fragmented state is obtained, with an extensive number of small groups. This is in contrast with the mean-field case in which the 


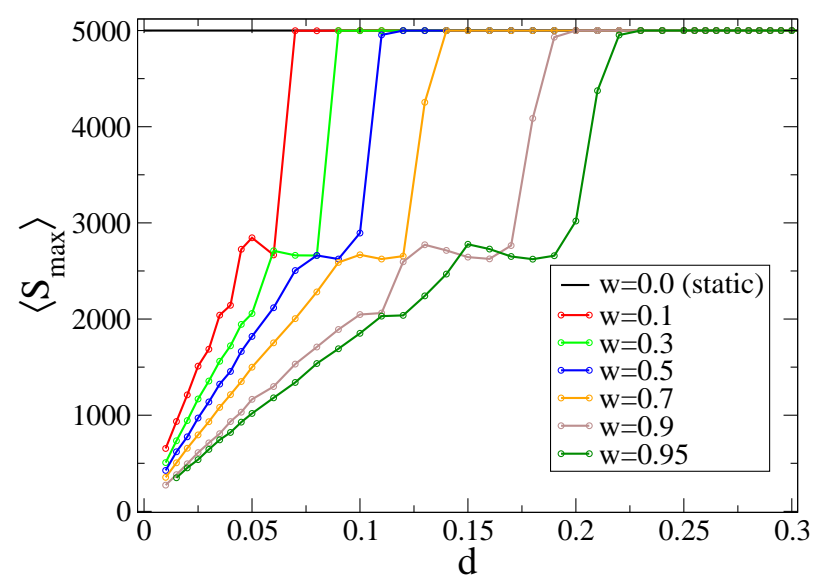

FIG. 14: (Color online) Maximal cluster size as a function of the tolerance of the agents for a variant of the model in which even agents with opinions outside of each other tolerance range can communicate with a probability given by Eq. (6).

number of groups is, roughly speaking, the inverse of the tolerance range.

When agents can rewire their links in a way depending on the opinions of their neighbors, i.e. break connections with neighbors with far apart opinions, the situation changes in various ways. At large tolerance values, the polarization transition is shifted since rewiring makes it easier for a large connected cluster to be broken in various parts. The possibility of network topological change therefore renders global consensus more difficult to achieve. On the other hand, for smaller tolerance values, the number of finite-size clusters is drastically reduced since agents can more easily find other agents with whom to reach an agreement. A real polarized phase is thus obtained, and the transition to a fragmented state is even suppressed: extensive clusters are obtained even at very low tolerance.

The detailed investigation of the system's time evolution reveals that the rewiring dynamics plays an important role both at early and late times: at earlytimes, adaptive rewiring enhances communication between agents and fosters giant cluster formation while, at late times, adaptation results in the breakup of the network into separate clusters after (or while) opinions evolve locally. The various involved timescales depend on the model's parameters and can be either well separated or similar.

Finally, we have considered changes in the microscopic rule of opinion evolution, from a strict and maybe unrealistic rule of sharp tolerance threshold to a smoother decrease of communication when opinions are further apart. Interestingly, such a change has a dramatic effect when the interaction network is fixed, since the system then always reaches consensus. The scenario of adaptive networks is however more robust, with a transition between consensus and polarized states as the tolerance is decreased. This emphasizes the relevance of considering the possibility of evolving topologies when studying the emergence of collective behavior in models for opinion formation.

Further investigations will consider the detailed topological structure of the clusters or groups of agents sharing the same opinion [37], and the evolution of other models for opinion dynamics on adaptive networks, where for example bounded confidence is either absent (such as the Voter model) or replaced by negotiation processes (such as the Naming Game). In such models, the asymmetry of the relation between the agents involved in an interaction has been shown to have further interesting effects on static networks [12, 28, 40], and can be expected to couple with the network evolution with new relevant consequences [41].
[1] J. Marro, P.L. Garrido, and M.A. Muñoz Eds., AIP Conf. Proc. 779, Granada, Spain (2005); W. Weidlich, Sociodynamics; A Systematic Approach to Mathematical Modelling in Social Sciences, Harwood Academic Publishers (2000); D. Stauffer et al. Biology, Sociology, Geology by Computational Physicists, Elsevier Science (2006).

[2] H. Föllmer, J. Math. Econ. 1, 51 (1974).

[3] C. Chamley, Rational Herds: Economic Models of Social Learning (Cambridge University Press, Cambridge, 2003).

[4] R. J. Glauber, J. Math. Phys. 4, 294 (1963).

[5] S. Galam, Y. Gefen, and Y. Shapir, Math. J. Soc. 9, 1 (1982).

[6] P. L. Krapivsky and S. Redner, Phys. Rev. Lett. 90, 238701 (2003).

[7] P. L. Krapivsky, Phys. Rev. A 45, 1067 (1992).

[8] R. Axelrod, J. of Conflict Resolut. 41, 203 (1997).

[9] L. Steels, Artificial Life Journal 2, 319 (1996).
[10] A. Baronchelli et al., J. Stat. Mech. P06014 (2006).

[11] A. Baronchelli, L. Dall'Asta, A. Barrat, and V. Loreto, Phys. Rev. E 73, 015102 (2006).

[12] L. Dall'Asta, A. Baronchelli, A. Barrat, and V. Loreto, Phys. Rev. E 74, 036105 (2006).

[13] X. Castelló, V. M. Eguíluz, and M. San Miguel, New J. Phys. 8, 308 (2006).

[14] G. Deffuant, D. Neau, F. Amblard, and G. Weisbuch, Advances in Complex Systems 3, 87 (2000).

[15] E. Ben-Naim, P. L. Krapivsky, and S. Redner, Physica D 183, 190 (2004).

[16] D. Stauffer and H. Meyer-Ortmanns, Int. J. Mod. Phys.C 15, 241 (2004).

[17] E. Ben-Naim, Europhys. Lett. 69, 671 (2005).

[18] F. Amblard and G. Deffuant, Physica A 343, 725 (2004).

[19] R. Pastor-Satorras and A. Vespignani, Evolution and structure of the Internet: A statistical physics approach (Cambridge University Press, Cambridge, 2004). 
[20] M. E. J. Newman, SIAM Review 45, 167 (2003).

[21] S. N. Dorogovtsev and J. F. F. Mendes, Evolution of networks: From biological nets to the Internet and $W W W$ (Oxford University Press, Oxford, 2003).

[22] S. N. Dorogovtsev and J. F. F. Mendes, Adv. Phys. 51, 1079 (2002).

[23] R. Albert and A.-L. Barabási, Rev. Mod. Phys. 74, 47 (2002).

[24] M. Granovetter, American Journal of Sociology 78, 1360 (1973)

[25] S. Wasserman and K. Faust, Social Network Analysis: Methods and applications (Cambridge University Press, Cambridge, 1994)

[26] C. Castellano et al., Phys. Rev. E 71, 066107 (2005).

[27] V. Sood and S. Redner, Phys. Rev. Lett. 94, 178701 (2005).

[28] K. Suchecki, V. M. Eguíluz, and M. San Miguel, Europhys. Lett. 69, 228 (2005).

[29] K. Suchecki, V. M. Eguíluz, and M. San Miguel, Phys. Rev. E 72, 036132 (2005).

[30] P. Holme and M. E. J. Newman, Phys. Rev. E 74, 056108 (2006).

[31] G. Caldarelli, A. Capocci, and D. Garlaschelli, arXiv.org cond-mat/0611201 (2006)

[32] D. Stauffer, M. Hohnisch, and S. Pittnauer, Physica A 370, 734 (2006).
[33] M. G. Zimmermann, V. M. Eguíluz, and M. San Miguel, Physical Review E 69, 65102 (2004).

[34] D. Centola, J. C. González-Avella, V. M. Eguíluz, and M. San Miguel, physics/0609213 (2006).

[35] S. Gil and D. H. Zanette, Physica D 224, 156 (2006).

[36] G. Ehrhardt, M. Marsili, and F. Vega-Redondo, International Journal of Game Theory 34, 383 (2006).

[37] A. Gautreau and B. Kozma, in preparation.

[38] E. Lopez, R. Parshani, R. Cohen, S. Carmi, S. Havlin, arXiv.org cond-mat/0702691 (2007).

[39] L. A. Braunstein, S.V. Buldyrev, R. Cohen, S. Havlin, and H. E. Stanley, Phys. Rev. Lett. 91, 168701 (2003).

[40] C. Castellano AIP Conf. Proc. 779, 114 (2005).

[41] C. Nardini, B. Kozma, and A. Barrat, in preparation.

[42] Other models consider that the new connection is established towards an agent with a similar opinion [30]; since this requires an a priori knowledge of the new agent's opinion, and in fact therefore of the whole system, we stick to a simpler hypothesis of a randomly established new connection.

[43] One can also think of a different rewiring rule in which $(i, j)$ is rewired to $(k, j)$. While the global qualitative picture does not change, the influence of this alternative rule deserves further investigation [37]. 